\title{
Advanced Dielectric Continuum Models of Solvation, Their Connection to Microscopic Solvent Models, and Application to Electron Transfer Reactions
}

\author{
I. V. Rostov*, M. V. Basilevsky ${ }^{\dagger}$, and M. D. Newton* \\ *Department of Chemistry, P. O. Box 5000, Brookhaven National Laboratory, Upton, NY 11973, USA: \\ ${ }^{\dagger}$ Karpov Institute of Physical Chemistry, Ul. Vorontsovo Pole 10, 103064, Moscow, Russia.
}

\begin{abstract}
Some recent advances in dielectric continuum models for static and dynamic aspects of molecular solvation are discussed, and connections with molecular-level solvent models are noted. The traditional Born-Onsager-Kirkwood (BKO) model is compared to a more flexible model (the so-called frequency-resolved cavity model (FRCM)) which assigns distinct inner and outer solute cavities in accommodating, respectively, the inertialess (optical) and inertial solvent response. Sample calculations of solvent reorganization energy $\left(\lambda_{s}\right)$ are presented for various thermal and optical electron transfer (ET) processes, based on self-consistent reaction field models using molecular orbital (MO) or configuration interaction (CI) solvent wave functions.
\end{abstract}

\section{INTRODUCTION}

In this chapter we survey recent solvation theories based on continuum models of polar solvents. The discussion includes a comparison of some refined continuum models with alternative molecular-level models. Practical applications are illustrated for electron transfer (EIT) reactions, specifically, for the evaluation of ET solvent reorganization energies, which represent an important nonequilibrium solvation phenomenon for which experimental data are available. Dynamic applications including time-dependent solvent Stokes shifts are also briefly mentioned.

The main idea inherent in a dielectric continuum model of a polar solvent is to introduce a dielectric permittivity as its basic characteristic. The Born theory of a solvated spherical ion comprises the simplest and most familiar case, dealing with static dielectric constant $\varepsilon_{0}$ as a single medium parameter. In advanced theories designed to provide some account of the internal structure and dynamics of the medium, the dielectric function $g(k, \omega)$ is introduced. This is a complex-valued quantity depending on wave vector $k$ and frequency $\omega$ [1]. The earlier pioneering implementations of this idea [2-5] neglected the important effect of excluded volume due to the solute particle. Incorporating it in a consistent theory requires, as shown

CP492, Simulation and Theory of Electrostatic Interactions in Solution, edited by Lawrence R. Pratt and Gerhard Hummer 1999 American Institute of Physics 1-56396-908-8

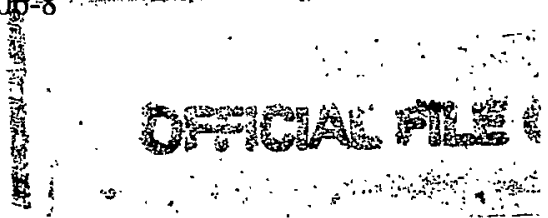


below, a significant extension of the original model. That is to say, the parameters determining the size and shape of a cavity, in which the solute particle is embedded according to the conventional continuum model, must be treated as dynamical variables on equal grounds with the electric field, a background variable of any continuum treatment. Only at such a sophisticated level a rational correspondence with molecular-level treatments of polar solvents can be gained.

Due to these cavity effects, the medium response function $K\left(\boldsymbol{r}, \boldsymbol{r}^{\prime}, \omega\right)$ (where $\boldsymbol{r}$ and $r^{\prime}$ are space point vectors) for full continuum dynamics becomes a property of the combined system, "solute + solvent", and its relation to the bulk solvent permittivity $\varepsilon(k, \omega)$ is very complicated. On the other hand, it is the response function (rather than $\boldsymbol{d}(\boldsymbol{k}, \omega))$ which is directly related to the microscopic statistics of solvation [6]. In this sense, a conceptual significance of $z(k, \omega)$ in solvation and ET problems [2-5] is reduced significantly.

Practical computation of equilibrium solvation effects is now readily available in terms of the polarized continuum model (PCM) [7, 8], which is also called BornKirkwood-Onsager (BKO) theory. It extends Born treatment of spherical ions to solutes with arbitrary shapes and charge distributions by directly solving the Poisson equation for a given charge distribution (actually, its integral equation counterpart $[9,10])$ with explicit account of matching conditions for relevant electric fields on the boundary of the solute cavity. By this means, the cavity effect is accurately incorporated at the static level. An extension of the PCM-BKO theory for treating nonequilibrium and dynamic solvation effects [10-15] underlies our presentation in Sections 2-4 where its recent developments are considered. For the sake of brevity, we omit discussion of dynamic effects of high-frequency inertialess polarization [14, 16]. Its time scale is considered to be infinitely fast (the so called Born-Oppenheimer approximation [14]) and the corresponding equilibrium contribution is included in all calculated solvation energies. In the BO model the fast solvent electrons "see" the instantaneous position of the transferring solute electron. In contrast, in the so-called SC (self consistent) model [14], the solvent electrons see the smeared out density of the transferring electron. For the purpose of evaluating solvent reorganization energy, the BO and SC models typically yield similar values, as in the cases exemplified in Section 6.

Although this unified treatment of solvation effects seems to result in a consistent theory, its straightforward further refinement meets serious and fundamental problems briefly mentioned above. They are discussed in Section 5 in comparison with related results of molecular simulation studies. Such studies (e.g., see [17]) are of great value in providing perspective in the parameters (generally based in large part on empirical fitting) which typically characterize continuum models.

In applications reported in Section 6, we discuss recent computations based on an extension of BKO theory denoted the "Frequency Resolved Cavity Model" (FRCM) [18]. This approach (described in Section 4 and the Appendix), which has been implemented in conjunction with semiempirical electronic structure models, seems to offer a useful compromise between ease of computation and flexibility of the underlying physical model, giving access to ET in complex molecular systems. 


\section{CONTINUUM MODEL FOR POLAR MEDIA IN THE FRAMEWORK OF LINEAR RESPONSE}

\section{Static theory}

Let us consider a solute particle with charge distribution $\rho(r)$ and a polar solvent with its polarization equilibrated to $\rho(\boldsymbol{r})$. When linear response pertains, the inertial potential field associated with the solvent polarization, $\Phi(\boldsymbol{r})$, is given by

$$
\Phi(r)=\hat{K} \rho(r)
$$

where $\hat{K}$ is a nonlocal linear operator,

$$
\hat{K} \rho(\boldsymbol{r})=\int d^{3} \boldsymbol{r}^{\prime} K\left(\boldsymbol{r}, \boldsymbol{r}^{\prime}\right) \rho\left(\boldsymbol{r}^{\prime}\right)
$$

Its kemel is a double-point symmetric function, $K\left(\boldsymbol{r}, \boldsymbol{r}^{\prime}\right)=K\left(\boldsymbol{r}^{\prime}, \boldsymbol{r}\right)$, known as Green's function in the theory of the potential [19]. A treatment of solvation and ET processes in terms of Eqs. (1) and (2) was introduced in Ref. [20].

In computational practice, these functional relations are implemented in terms of a basis set expansion, which is conveniently based on a configuration interaction (CI) expansion of the solute wave function $\psi[10-12]$ :

$$
\psi=\sum_{a} C_{a} D_{u} ; \quad a=1, \ldots, N
$$

where the $D_{a}$ are properly chosen orthonormal Slater determinants. The corresponding charge density expansion is

$$
\rho(\boldsymbol{r})=\sum_{a b} C_{a} C_{b} \rho_{a b}(\boldsymbol{r})
$$

where the $\rho_{a b}$ are first order transition density matrix comprised of the respective elements of the first order density matrix in the determinantal basis (the electron density component). The diagonal elements $\left(\rho_{a a}\right)$ also include the contributions from the nuclear (core) charges. The $\mathrm{Cl}$ secular equation reads

$$
\begin{gathered}
\left.\left.\dot{H}\left|C^{(k)}\right\rangle\right\rangle=W_{k}\left|C^{(k)}\right\rangle\right\rangle \\
\left.\left|C^{(k)}\right\rangle\right\rangle=\left(\begin{array}{c}
C_{1}^{(k)} \\
\vdots \\
C_{N}^{(k)}
\end{array}\right)
\end{gathered}
$$


The elements of the Hamiltonian matrix $H$

$$
H_{u b}=H_{a b}^{(0)}+Y_{a b}
$$

contain the gas phase component modified (in the case of the BO limit $[12,14]$ ) by the fully equilibrated inertialess (high-frequency) polarization (the first term) and matrix elements of the inertial (low-frequency response field

$$
Y_{a b}=\int \mathrm{d}^{3} \boldsymbol{r} \rho_{a b} \Phi
$$

The eigenvalues $W_{k}$ and eigenvectors $\left.\left|C^{(k)}\right\rangle\right\rangle$ of Eq. (5) depend parametrically on $Y_{a b}$. The set of $Y_{a b}(a \geq b)$ can be considered as medium coordinates [10-12].

The matrix counterpart of the Green's function $K\left(r, r^{\prime}\right)$ is the reorganization matrix $T$ with elements

$$
\begin{aligned}
& T_{u b, c d}=-\int d^{3} r \rho_{c b} \hat{K} \rho_{c d}=-\int d^{3} r \rho_{u b} \Phi_{c d} \\
& T_{a b, c d}=T_{c d, u b} \\
& \text { where } \Phi_{c d d} \equiv \hat{K} \quad \rho_{c d}
\end{aligned}
$$

The $T$ matrix elements can be used to define "inertial" solvation energies; e.g.,

$$
U_{a b, a b}=-\frac{1}{2} T_{a b, a b}
$$

represents the equilibrium inertial solvation free energy associated with charge density $\rho_{a b}$.

The medium polarization self-energy $S$ is a quadratic form of medium coordinates [12]:

$$
S=\frac{1}{2} \sum_{\substack{c b, c a \\ a \geq b, c z d}}\left(T^{-1}\right)_{a b, c d} Y_{u b} Y_{c d}
$$

The total free energy surface is expressed in terms of $Y_{a b}$ as

$$
U_{k}\left(Y_{a b}\right)=S\left(Y_{a b}\right)+W_{k}\left(Y_{a b}\right)
$$

The form of $S$ emphasizes the fundamental importance of the reorganization matrix. 


\section{Dynamic Theory}

A natural time-dependent extension of the linear response relation (1) is

$$
\begin{aligned}
& \Phi(\boldsymbol{r}, t)=\hat{K} \rho(\boldsymbol{r}, t) \\
& \hat{K} \rho(\boldsymbol{r}, t)=\int d t \int d^{3} \boldsymbol{r}^{\prime} K\left(\boldsymbol{r}, \boldsymbol{r}^{\prime}, t-t^{\prime}\right) \rho\left(\boldsymbol{r}^{\prime}, t^{\prime}\right)
\end{aligned}
$$

Its treatment is most convenient in terms of frequency domain $(\omega)$ Fourier transforms [1]. Let us consider the expansion

$$
\rho(r, \omega)=\sum \rho_{a b}(r) m_{a b}(\omega)
$$

where $m_{a b}(\omega)$ are the expansion coefficients determined by conditions of a timedependent experiment; two illustrations are given below. A full analogue of the static relation (1) is

$$
\Phi(\boldsymbol{r}, \omega)=\hat{K}(\omega) \rho(\boldsymbol{r}, \omega)
$$

where $\Phi(r, \omega)$ and $\rho(r, \omega)$ are the Fourier transforms, respectively, of $\Phi(r, t)$ and $\rho(r, t)$, and the operator $\hat{K}(\omega)$ is defined in terms of its kernel, the complex-valued doublepoint function $K\left(r, r^{\prime}, \omega\right)$ obtained as a transform of the kernel $K\left(r, r^{\prime}, t-t^{\prime}\right)$. A matrix counterpart of the linear response relation (14) is given by

$$
\left.\left.\left|Y_{a b}(\omega)\right\rangle\right\rangle=-T(\omega)\left|m_{a b}(\omega)\right\rangle\right\rangle
$$

Here, as usual, the $|\ldots\rangle\rangle$ denote column-vectors. $Y_{a b}(\omega)$ are the Fourier transforms of the time-dependent medium variables $Y_{a b}(t)$, and the reorganization matrix $T(\omega)$ is calculated according to the prescription (8) with the kernel $K\left(r, r^{\prime}, \omega\right)$.

\section{First Example: The Stokes Shift}

The time-dependent solvent stokes shift is observed when the charge distribution changes instantaneously:

$$
\rho(\boldsymbol{r}, t)=\rho_{0}(\boldsymbol{r})+\Theta(t) \Delta \rho(\boldsymbol{r})
$$

Notations $\rho_{0}(\boldsymbol{r})$ and $\Delta \rho(\boldsymbol{r})$ are obvious, $\Theta(t)$ represents the step function. In this case $m_{a b}(\omega)$ are expressed in terms of the Fourier transform of $\Theta(t)$. Implementations of Eq. (16) to treat Stokes shifts are well known [21,22]; the above technique [23-25] opens the way for straightforward PCM-BKO computations for solutes with arbitrary shapes and charge distributions. 
Second example: The equation of motion for $Y_{a b}(t)$

We derive first the expression for expansion coefficients $m_{a b}$ in a system "solute + solvent" without external forces. Consider function $W\left(Y_{a b}\right)$, a solute contribution to the FES $U\left(Y_{a b}\right)$, Eq. (11). (The index labeling an energy level is omitted hereafter). According to the Hellman-Feynman theorem, $\rho=\frac{\delta W}{\delta \Phi}$ (a functional derivative) [10]. From Eq. (7) we conclude that

$\rho=\sum \frac{\partial W}{\partial Y_{a b}} \frac{\delta Y_{a b}}{\delta \Phi}=\sum \frac{\partial W}{\partial Y_{a b}} \rho_{a b}$

Thus

$$
m_{a b}(\omega)=\frac{\partial W}{\partial Y_{a b}}(\omega)
$$

where the term on the right is the Fourier transform of the time-dependent derivative $\frac{\partial W}{\partial Y_{a b}}(t)$.

The dynamic equation can now be written as

$$
\left.\left.\left.T^{-1}(\omega)|Y(\omega)\rangle\right\rangle=-\left|\frac{\partial W}{\partial Y}(\omega)\right\rangle\right\rangle+|\operatorname{GRF}\rangle\right\rangle
$$

(indices " $a b$ " are omitted). This is identical to Eq. (15) supplemented by the vector of Gaussian random forces $(\mathrm{GRF}(\omega))$ in the $\omega$-representation. It is connected with the generalized susceptibility matrix $T^{-1}(\omega)$ via the fluctuation-dissipation theorem. Random forces introduce fluctuations in the dynamics of medium coordinates.

An inverse Fourier transform of Eq. (18) is available provided we find, at a static level, a stationary point $|\bar{Y}\rangle\rangle$ of the FES $U$, Eq. (11), satisfying the relation $(T(\omega=0)=T$, the static case):

$$
\left.\left.T^{-1}|Y\rangle\right\rangle=-\left|\frac{\partial W}{\partial Y}\right\rangle\right\rangle
$$

The regular forces can be linearized in its vicinity:

$$
\left.\left.\left.\left.\left.\left|\frac{\partial W}{\partial Y}\right\rangle\right\rangle=T^{-1}|Y\rangle\right\rangle+\Lambda(Y\rangle\right\rangle-|\bar{Y}\rangle\right\rangle\right)
$$


In this approximation, the stochastic equation of motion for variables $|\Delta Y(t)\rangle\rangle=|Y(t)\rangle\rangle-|\bar{Y}\rangle\rangle$ is recovered in the time domain [26]. The dynamical evolution is governed by the operator $\left(\hat{T}^{-1}-T^{-1}\right)$ where $\left.\left.\hat{T}^{-1}|\Delta Y(t)\rangle\right\rangle=\int_{-\infty}^{t} T^{-1}\left(t-t^{\prime}\right)\left|\Delta Y\left(t^{\prime}\right)\right\rangle\right\rangle d t^{\prime}$, yielding the following set of generalized Langevin equations,

$$
\left.\left.\left.\left(\hat{T}^{-1}-T^{-1}\right)|\Delta Y\rangle\right\rangle=-\left(T^{-1}+\Lambda\right)|\Delta Y\rangle\right\rangle+|\mathrm{GRF}\rangle\right\rangle
$$

The random forces here are in the time domain and the matrix of force constants in the regular force is composed of solvent $\left(T^{-1}\right)$ and mixed solute-solvent $(\Lambda)$ components (note that the sign convention for $T$ in [23] is opposite to that employed here and elsewhere $[12,18]$ ).

By this means, the computation of matrix $T(\omega)$ becomes a key procedure in dynamical implementations. As applied to adiabatic ET reactions, the subsequent rate calculation for Eq. (21) is available in terms of the Kramers-Grote-Hynes (KGH) approximation $[27,28]$. Such computations for model ET systems with a single medium coordinate have been reported [23].

\section{METHODS FOR CALCULATION OF THE REORGANIZATION MATRIX AND THE SOLVATION ENERGY}

The formal scheme as presented in Section 2 furnishes a general approach for treatment of equilibrium and nonequilibrium solvation effects in polar media. This formulation is independent of the particular computational method invoked to perform the linear response relations like Eqs. (1) or (14). The basic quantity of this theory is the reorganization matrix $T$, generally $\omega$-dependent, and we focus now on practical methodologies for its computation. Solute excluded volume must be necessarily taken into consideration in such a treatment and the simplest electrostatic continuum model to treat operator $\hat{K}$ at such a theoretical level is the PCM-BKO theory $[7,8]$. (Simplified treatments like COSMO [29], generalized Born approach [30] and others involving different approximations in order to avoid an explicit treatment of boundary effects [31] could also be employed in this context). The PCMBKO approach expresses the response field $\Phi(\boldsymbol{r})$ in terms of the surface charge density $\sigma\left(r^{\prime}\right)$ on the boundary surface $\Sigma$,

$$
\Phi(r)=\int d^{2} r^{\prime} \frac{\sigma\left(r^{\prime}\right)}{\left|r-r^{\prime}\right|}
$$


and $\sigma$ is extracted as a solution to the relevant electrostatic integral equation based on the solute charge density $\rho[10]$. Because we consider $\Phi(\boldsymbol{r})$ as an inertial component of the total response field, $\sigma$ is also an inertial quantity

$$
\sigma\left(\boldsymbol{r}^{\prime}\right)=\sigma_{\mathrm{tot}}\left(\boldsymbol{r}^{\prime}\right)-\sigma_{\infty}\left(\boldsymbol{r}^{\prime}\right)
$$

The surface densities $\sigma_{\text {tot }}\left(\boldsymbol{r}^{\prime}\right)$ (total) and $\sigma_{\infty}\left(\boldsymbol{r}^{\prime}\right)$ (inertialess) are calculated by solving the PCM-BKO equation twice: first, with static dielectric permittivity $\varepsilon=\varepsilon_{0}$ (for $\sigma_{\mathrm{tot}}$ ) and, second, with optical permittivity $\varepsilon=\varepsilon_{\infty}$ (for $\sigma_{\infty}$ ). The desired elements of the static $(\omega=0)$ reorganization matrix can then be obtained according to Eq. (8). If the initial and final states in a two-state ET process correspond. respectively, to charge densities $\rho_{1}$ and $\rho_{f}$, then the solvent reorganization energy $\lambda_{s}$ may be expressed as $[12,18]$ :

$$
\lambda_{3}=\frac{1}{2} T_{t, t f}
$$

where $\rho_{i f} \equiv \rho_{f}-\rho_{i}$. For an optical ET process, $\lambda_{s}$ may be identified with one-half of the solvent contribution to the Stokes shift [32,33]. Calculations of $\lambda_{s}$ have been reported both for model [12.32] and experimentally studied [33] ET systems. The PCM-BKO computations of the dynamic $\omega$-dependent solvation energies have appeared recently [23-25] and compared with observed time-dependent Stokes shifts. (note that the original technique developed in [24] is equivalent to the $\omega$-dependent PCM-BKO technique $[23,25])$.

More sophisticated continuum approaches include the double-cavity (the FRCM scheme [18]) and the nonlocal electrostatic [34] treatments; their tentative application to the Stokes Shift kinetics has also been tested [25].

A merit of the formulation given in Section 2 is that it applies equally well to molecular-level treatment of the response field $\Phi(\boldsymbol{r})$. There is no constraint on the computational treatment of operator $\hat{K}$ in Eqs. (1), (12) or (14) except an obvious condition that it is linear. Integral equations based on the statistical theory of liquids (the modified RISM scheme [35]) have been applied to calculate solvent reorganization energies over a wide range of solvent polarity [36]. It would also be of interest to implement Eqs. (1) and (12) using molecular dynamics (MD) simulation techniques.

\section{THE FRCM APPROACH}

The PCM-BKO treatment of reorganization energies for several intramolecular ET reactions proved to be unsatisfactory [33]. When a standard parameterization of the cavity size. fitted to reproduce equilibrium solvation energies, was applied, the computed reorganization energies (non-equilibrium quantities) were found to be greatly in excess of experimental estimates. It was concluded that the 
PCM-BKO approach failed to reproduce both equilibrium and nonequilibrium solvation effects within the same parameterization scheme.

The frequency resolved cavity model (FRCM [18]) was elaborated in order to circumvent this deficiency. This method differs from the PCM-BKO method by separating the inertialess (high-frequency) response of the medium from the inertial (low-frequency) one. The solute is surrounded by two surfaces $\left(\Sigma_{1}\right.$ and $\left.\Sigma_{2}\right)$, each of which is constructed as a collection of overlapping spheres similar to the PCM-BKO model. For the first cavity, contained within the internal surface $\Sigma_{1}$, the radius of each sphere is defined as $r_{1}=\kappa r_{\mathrm{vdw}}$, where $\kappa=0.9$ is a universal empirical factor common to all solvents and $r_{\mathrm{vdW}}$ is the van der Waals radius of the particular solute atom. The radius $r_{2}$ defining the external surface $\Sigma_{2}$ is given by

$$
r_{2}=r_{1}+\delta=0.9 r_{\mathrm{vdW}}+\delta
$$

where $\delta$ is another empirical constant, pertaining to the given solvent (it correlates roughly with the characteristic size of a solvent particle). Between the two surfaces the medium is represented by the inertialess dielectric constant $\varepsilon_{\infty}$, while outside the outer cavity the static dielectric constant $\varepsilon_{0}$ is applied. The layer between the surfaces corresponds roughly to the first solvation shell. Calculation of the potential field $\Phi(r)$ in this scheme amounts to simultaneously solving two equations describing charge densities on the two cavities, namely $\sigma_{1}$ (on the inner surface) and $\sigma_{2}$ (on the outer one); they contribute additively to the total $\Phi(\boldsymbol{r})$ in terms of two equations similar to Eq. (22). More details about the FRCM technique, including the formulation of the inertial field component, are given in the Appendix.

A simple illustration of this approach is a spherically symmetric Born-like case (a point ion of charge $Q$ placed at the center of two concentric spheres of radii $r_{1}$ and $r_{2}$ ). The total solvation energy $[18,37]$,

$$
U_{\text {solv }}=-\frac{Q^{2}}{2}\left[\left(1-\frac{1}{\varepsilon_{\infty}}\right) \frac{1}{r_{1}}+\left(\frac{1}{\varepsilon_{\infty}}-\frac{1}{\varepsilon_{0}}\right) \frac{1}{r_{2}}\right]
$$

is naturally divided into inertialess and inertial contributions; it reduces to the Born expression when $r_{1}=r_{2}$. The extra cavity parameter, namely the width $\delta$ of the intersurface layer in Eq. (25), brings additional flexibility in accounting simultaneously for both equilibrium solvation energies and the nonequilibrium ET reorganization energies [18] (see also section 6).

\section{A CONNECTION TO MICROSCOPIC TREATMENTS OF SOLVENT STRUCTURE}

A nonlocal electrostatic theory $[38,39]$ is a conventional way to bring elements of internal solvent structure into a continuum solvent model. In the static 
case, the dielectric function $g(k)$ carries all the information about the solvent structure. Its simplest Lorentzian version is

$$
\varepsilon(k)=\varepsilon_{\infty}+\frac{\varepsilon_{0}-\varepsilon_{x}}{1+(\lambda k)^{2}}
$$

where the parameter $\lambda$ is called "the correlation length" and is interpreted as a measure of size of a solvent particle. More detailed description with several Lorentzian terms can be also considered [39]. In real space, the inverse Fourier transform of $\varepsilon(k)$, namely the nonlocal kernel $\varepsilon\left(\boldsymbol{r}-\boldsymbol{r}^{\prime}\right)$, is an exponentially decreasing function of distance $\left|\boldsymbol{r}-\boldsymbol{r}^{\prime}\right|$.

For a spherical ion of radius $r_{1}$ the expression for solvation energy is available either in terms of an approximate nonlocal approach in which the treatment of the excluded volume effect is incomplete [38, 39] or by an accurate treatment of matching conditions on the boundary of the spherical cavity [40]. In both theories this expression depends on the parameter

$$
\eta=\frac{\lambda}{r_{1}}
$$

which is a ratio of characteristic lengths of solvent and solute particles. It is not possible to find such a relationship in local theories, where $\eta=0$. On the other hand. even in simple molecular theories based on the "mean spherical approximation" (MSA) [41]this ratio appears naturally, with the solvent particle modeled as a hard sphere having radius $\lambda$.

Recent studies based on microscopic molecular solvent models [42] have brought into doubt the validity of Lorentzian model for a dielectric function $\boldsymbol{g}(\boldsymbol{k})$, revealing that it has a pole structure with terms proportional to $\left[1-(\lambda k)^{2}\right]^{-1}$. In realspace this leads to an oscillatory behavior of the nonlocal kernel $\varepsilon\left(\left|\boldsymbol{r}-\boldsymbol{r}^{\prime}\right|\right)$. When inserted in a nonlocal theory, such oscillating kernels result in an irregular behavior of the solvation energy as a function of ion radius $r_{1}[40,43]$. This problem has been eliminated [40] by employing a variational solvation model. in which the variational free energy functional includes as variables both the solvent polarization field and the cavity radius on an equal footing(this idea has been independently elaborated [44] in a different way). Smooth solvation curves were obtained by this technique.

The experience gained by studying these advanced models draws to the following conclusions [40]. The addition of pole terms to a Lorentzian dielectric function violates the positive definiteness of the integral operator goveming the basic equation of a nonlocal theory with excluded volume. Modifications of the cavity model tend to restore the correct properties of the integral kernel. or, at the very least, serve to approach them as closely as possible. On the basis of simple physical reasoning, we observe that oscillations introduced by the poles of $\boldsymbol{a}(\boldsymbol{k})$, related to real spatial correlations inside a solvent, are incompatible with the frozen cavity model, in 
which the radius $r_{1}$ is considered as an intrinsic property of a given solute ion. The above modifications of the cavity model introduce more flexibility by allowing for the mutual adjustment of the cavity size and solvent structure in the vicinity of the ion.

For practical applications this notion suggests that simple Lorentzian models of $\varepsilon(k)$, in which the above-mentioned instabilities are absent (the corresponding Lorentzian integral kernels are positive-definite), seem to have a rational justification and may serve as a basis for a semiempirical continuum solvation theory like the FRCM model.

A complicated oscillatory behavior of a dielectric function in real liquids arises due to the interference of polarization and density fluctuations [45]. In the vicinity of the solute, according to the mechanism described above, the fluctuations of the cavity are strongly correlated with the solvent density fluctuations. To a significant extent, these fluctuations cancel each other, leaving the orientational polarization as the dominant in the effective solvent field. By this means (returning to the notation of section 2) we may infer that an effective Lorentzian dielectric function mimics the response function $K\left(r, r^{\prime}, \omega\right)$ (or matrix $T(\omega)$ ), a property of a combined system ("solute + solvent") rather than the bulk permittivity $\varepsilon(k, \omega)$, which represents the pure solvent and displays a much more complicated behavior in real space.

We conclude that the idea of a cavity possessing a definite shape and a fixed boundary surface seems to be the weakest point of continuum solvent models. Its consistent elaboration in terms of compensating fluctuations needs a very sophisticated theory, which would not likely be practical. The corresponding higher order effects, to the extent that they are considered important, are better addressed in terms of molecular simulations.

\section{SAMPLE CALCULATIONS OF SOLVENT REORGANIZATION ENERGY}

Earlier work [18] has demonstrated the ability of the FRCM scheme to describe simultaneously both equilibrium (solvation energies) and non-equilibrium (ET reorganization energies) solvation effects after a proper choice of parameters in Eq. (25) was made. Both methods of treatment of the inertialess polarization (either the $\mathrm{SC}$ or the $\mathrm{BO}$ approximation) resulted in practically the same values for solvent reorganization energies.

As an illustration of the application of the above techniques, we now report the evaluation of the solvent reorganization energy $\left(\lambda_{5}\right)$ for intramolecular ET involving both ground and electronically excited states of some donor-bridgeacceptor (DBA) systems. The results shown below were chosen to exemplify the sensitivity of $\lambda_{s}$ magnitude to various features of the DBA system or the continuum model adopted. Some comparison with experiment is also provided. 


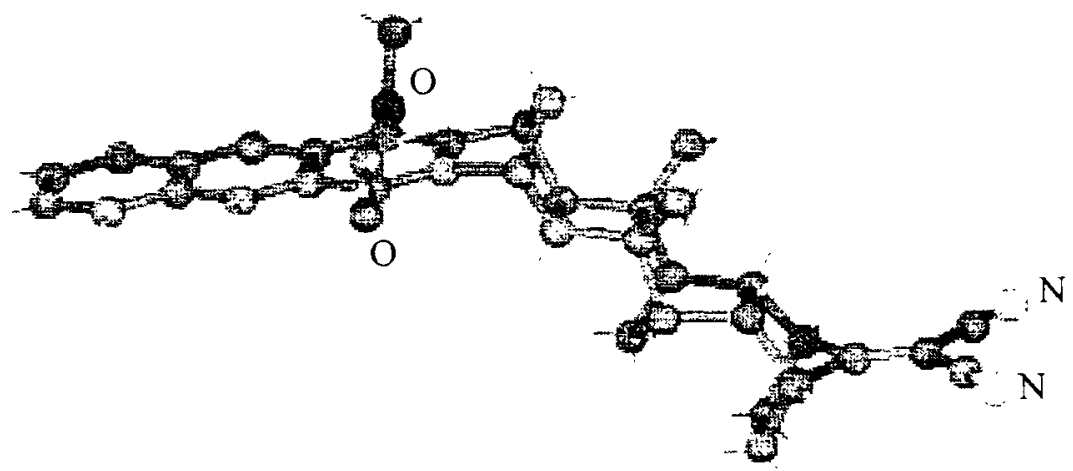

$\mathrm{I} a$

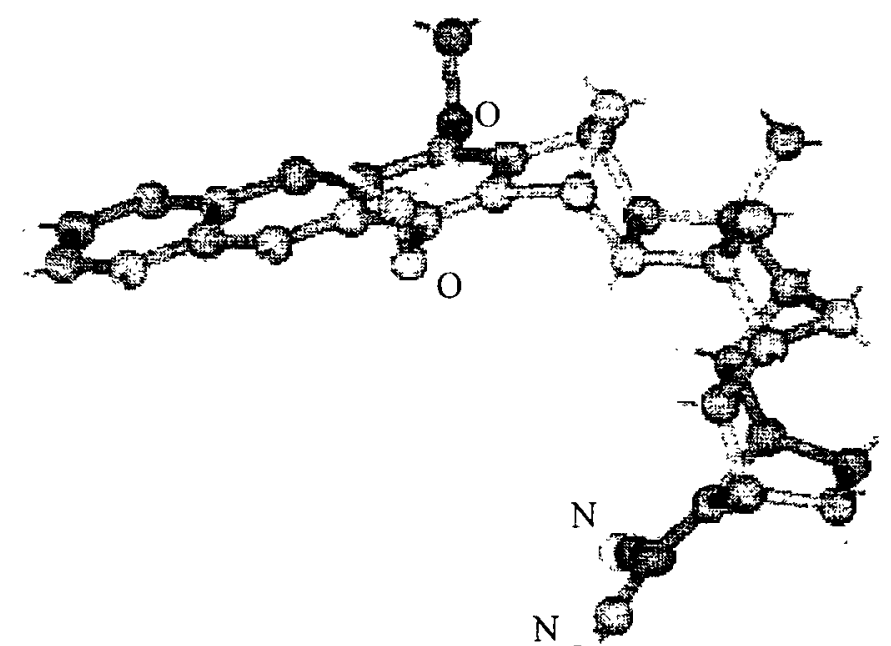

$\mathrm{I} b$

Figure 1. Structures of Series I. Unlabelled large dark (small light) spheres denote, respectively $C$ and $\mathrm{H}$ atoms. The $\mathrm{D}$ and $\mathrm{A}$ groups are, respectively, the dimethoxy (left hand side) and dicyano vinyl (right hand side) moieties. See also [46].

\section{Variation of $\lambda_{s}$ with D/A separation}

Table 1 illustrates the pronounced dependence of $\lambda_{s}$, for a given $\mathrm{D} / \mathrm{A}$ pair on the effective distance separating the $\mathrm{D}$ and $A$ sites, as expected from the simple 2sphere Marcus model $[5,18]$. The calculated dipole moment shift seems to yield a distance very similar to that based on the center-to-center separation. For both systems ( $a$ and $\mathrm{I} b$ in Fig. 1) the quantum calculations yield essentially identical results for the forward photoinitiated charge separation $\left(\mathrm{CS}: \mathrm{D}^{*} \mathrm{BA} \rightarrow \mathrm{D}^{+} \mathrm{BA}^{-}\right.$) and 
TABLE 1. Calculated $\lambda_{s}$ for ET in $\mathrm{l} a$ and $\mathrm{l} b$ in THF solvent ${ }^{\mathrm{a} b}$ : Dependence on D/A Separation $\left(r_{\mathrm{DA}}\right)$.

\begin{tabular}{cccc}
\hline Structure & $\lambda_{s}(\mathrm{eV})$ & $\Delta \mu / e(\AA)^{\mathrm{c}}$ & $r_{\mathrm{DA}}(\AA)^{\mathrm{d}}$ \\
\hline $\mathrm{I} a$ & 0.49 & 12.1 & 12.9 \\
$\mathrm{I} b$ & 0.33 & 8.3 & 8.4 \\
\hline
\end{tabular}

a To the precision displayed the same results were obtained for CS and CR in each system (Ia and Ib).

bRCM calculations based on configuration interaction (CI) employing single excitations involving the highest five occupied and lowest five unoccupied MO's of the ground state self consistent field (SCF) wavefunction, using the AM1 Hamiltonian [47] at the SC level (see Section 1) and dielectric constants given in [48].

${ }^{c}$ Calculated shift in state dipole moment for CS and CR processes.

${ }^{d}$ Center-to-center D/A separation.

back charge recombination (CR: $\mathrm{D}^{+} \mathrm{BA}^{-} \rightarrow \mathrm{DBA}$ ) processes. In general, $\mathrm{CS}$ and $\mathrm{CR}$ processes may be expected to yield distinct $\lambda_{s}$ and $\Delta \mu$ values.

\section{Variation of $\lambda_{s}$ with $\mathbf{D} / \mathrm{A}$ type}

Table 2 shows the appreciable variation in $\lambda_{s}$ and $\Delta \mu$ associated with six different combinations of aromatic $\mathrm{D}$ and $\mathrm{A}$ groups for a given bridge moiety (Fig. 2). Once again (see Table 1) an inverse relationship between $\lambda_{s}$ and D/A separation is displayed, with similar estimates of the latter based on $\Delta \mu / e$ and center-to-center distance $\left(r_{\mathrm{DA}}\right)$. The calculated and experimental results [49] are seen to be in close accord.

TABLE 2. Comparison of Calculated and Experimental $\lambda_{s}$ Values for Charge Shift ET $\left(\mathrm{D}^{\top} \mathrm{BA} \rightarrow \mathrm{DBA}^{-}\right)$in Series II in THF Solvent

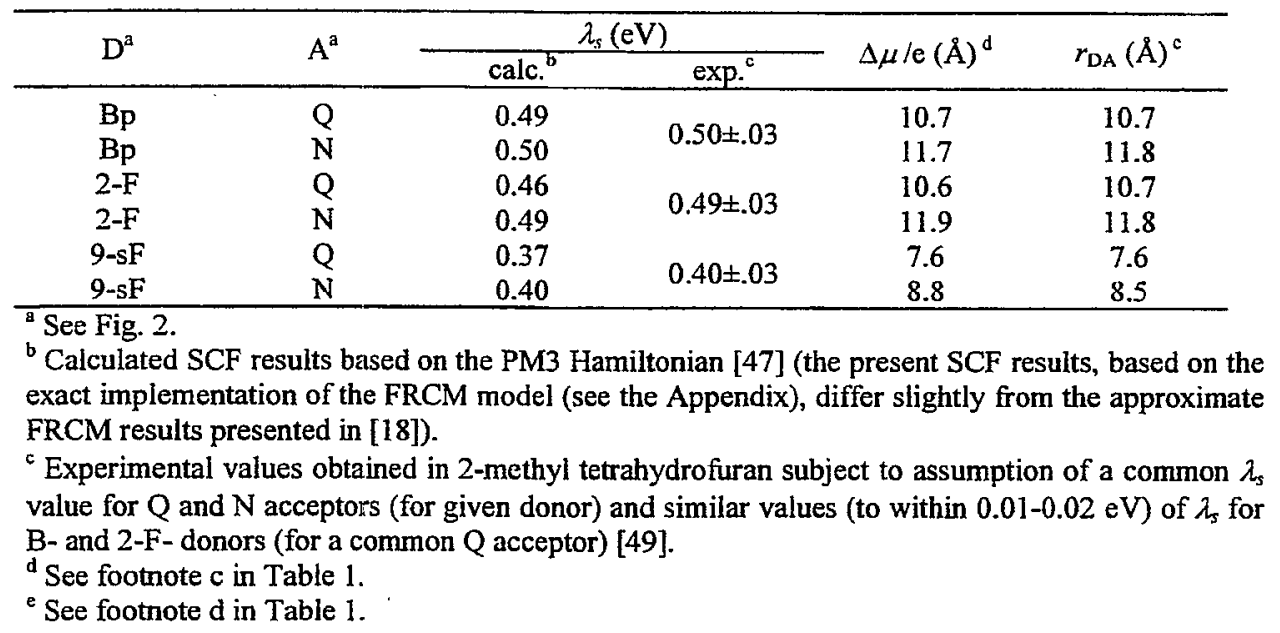





Figure 2. Molecular fragments serving as donor (left), bridge (center), or acceptor (right) groups in structures of Series II.

\section{Sensitivity of $\lambda_{s}$ to Continuum Model}

Table 3 shows the considerable difference in $\lambda_{s}$ magnitude based on the FRCM model [18] and the conventional PCM-BKO model. $[8,10]$. Furthermore, failure to impose full treatment of dielectric boundary conditions is seen to cause error of up to $\sim 20 \%$ for the case shown $(E T$ with $D=B p$ and $A=N)$.

TABLE 3. Dependence of $\lambda_{s}$ magnitude on continuum model for the charge shift ET process in Series II $(D=B p, A=N)$ in THF

\begin{tabular}{ccc}
\hline \multicolumn{2}{c}{ Continuum model } & $\lambda_{s}(\mathrm{eV})$ \\
\hline FRCM $^{\mathrm{a}}:$ & full $^{\mathrm{b}}$ & 0.48 \\
& ${\text { neglect of } S_{12} \text { term }^{\mathrm{c}}}^{\mathrm{c}}$ & 0.46 \\
PCM-BKO & uniform $^{\mathrm{d}}$ & 0.37 \\
& full $^{\mathrm{b}}$ & 1.21 \\
& uniform $^{\mathrm{d}}$ & 0.98 \\
\hline
\end{tabular}

${ }^{a}$ See Fig 2 and footnote $b$ in Table $2 .{ }^{b}$ Full treatment of dielectric boundary conditions.

bull treatment of dielectric boundary conditions.

' See Appendix.

Approximate treatment of dielectric boundary conditions [18]. 


\section{Variation of $\lambda_{s}$ with temperature}

The extent of temperature variation of $\lambda_{s}$, based both on theoretical models and experimental results, has been the subject of recent investigations [50-52]. While the temperature dependence of dielectric constants $\left(\varepsilon_{0}\right.$ and $\left.\varepsilon_{\infty}\right)$ implies a positive temperature derivative $\lambda_{s}$ for moderate to strongly polar solvents, based on PCMBKO or FRCM models, recent theoretical analysis [50] points to the dominant role of density fluctuation in leading to a negative temperature derivative, in conformity with inferences based on recent experimental studies $[51,52]$. In a preliminary attempt to account for such a negative slope within the framework of the FRCM model, we consider the following expression,

$$
\frac{d \lambda_{s}}{d T}=\left(\frac{\partial \lambda_{s}}{\partial T}\right)_{\delta}+\left(\frac{\partial \lambda_{s}}{\partial \delta}\right)_{T} \frac{d \delta}{d T}
$$

where the first term on the right hand side includes the temperature dependence of $\varepsilon_{0}$ and $\varepsilon_{\infty}$, and where $\delta$ is the parameter characterizing the solvent-dependent radius controlling the inertial response in the FRCM model [18] (Eq. (25)). Table 4 offers an example based on ET in species III (DBA $\left.\rightarrow \mathrm{D}^{+} \mathrm{BA}^{-}\right)$

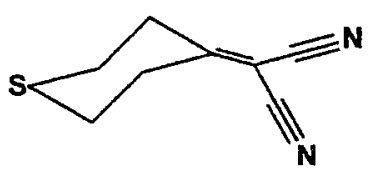

III

where $\mathrm{D}$ and $\mathrm{A}$ refer to the thio and dicyanovinyl moieties) [52]. For this process a value of $d \delta d T=0.0042 \AA / \mathrm{K}$ is found to reproduce the experimental value of $d \lambda_{s} / d T$

TABLE 4. Modelling temperature variation of $\lambda_{s}$ in terms of temperature dependent $\delta$ parameter: optical ET in III $^{\mathrm{a}}$ in acetonitrile.

\begin{tabular}{|c|c|c|c|}
\hline \multicolumn{2}{|c|}{ calc. ${ }^{b}$} & \multicolumn{2}{|c|}{ exp. $^{c}$} \\
\hline$\lambda_{s}(\mathrm{eV})$ & $d \delta / d T(\AA / \mathrm{K})$ & $\lambda_{s}(\mathrm{eV})$ & $d \lambda_{s} / d T\left(10^{-3} \mathrm{eV} / \mathrm{K}\right)$ \\
\hline 0.35 & $0.0042^{\mathrm{d}}$ & $0.52^{e}$ & -0.77 \\
\hline
\end{tabular}

${ }^{\mathrm{a}} \mathrm{DBA} \rightarrow \mathrm{D}^{\top} \mathrm{BA}^{-}$, where in III, the $\mathrm{D}$ and $\mathrm{A}$ sites are localized respectively, in the vicinity of the thio and dicyanovinyl moieties.

${ }^{b}$ Based on Cl calculation using AM1 Hamiltonian [47].

c Obtained from combined use of absorption and emission data [52].

d Obtained from a quadratic fit to results obtained for a finite grid of $T(298 \pm 20 \mathrm{~K})$ and $\delta$ $(1.8 \pm 0.05 \AA)$ values.

Obtained by subtracting an estimated molecular contribution $(0.45 \mathrm{eV})$ from total reorganization energy [52]. 
when combined with the other quantities in Eq. (29), which are obtained from FRCM configuration interaction $(\mathrm{CI})$ calculations. A connection of this phenomenological interpretation of the temperature trend in $\lambda_{s}$ to a molecular theory can be recognized from the fact that $\delta$ is closely related to an effective correlation length associated with the size of solvent particles (Section 5). This correlation length is strongly influenced by temperature-dependent fluctuations of the density and orientation of the solvent molecules. See also the work on aqueous acid reported in [53], which implied a small increase of effective solute radius with temperature. For related discussion of cavity fluctuations see [44].

\section{DISCUSSION}

The examples in the previous section demonstrate the versatility of the combined, self-consistent use of flexible quantum chemical and reaction filed techniques in addressing ET processes for a variety of chemical situations, including different types of solvents, donor/acceptor pairs, and electronic states (both ground and excited). A preliminary attempt to model the temperature dependence of $\lambda_{s}$ has been made, based on the idea of a temperature dependent cavity radius. All of these examples, together with the discussion given in Section 5 , make clear the importance of systematic efforts to employ suitably designed molecular level simulations as a mean of evaluating the parameters defining continuum solvation models (e.g., see [17]). In this manner, one may hope to reduce the level of empiricism characteristic of continuum models in use at present, leading to a rational basis for formulating a molecular cavity as a joint property of a given solute-solvent pair, taking due account of factors such as the charge and electronic state of the solute species, the role of different frequency domains of the medium response, and the temperature and pressure of the system.

\section{ACKNOWLEDGEMENTS}

The work at Brookhaven National Laboratory was supported by the Division of Chemical Sciences, U. S. Department of Energy, under grant DE-AC0298CH10886. The work of Russian side was supported in part by the Russian Foundation for Basic Research (RFBR) and the International Association for the promotion of co-operation with scientists from the New Independent States of the former Soviet Union (INTAS) (RFBR grants 96-15-97465, 99-03-33196, INTASRFBR grant IR-97-620). We are grateful to Prof. M. B. Zimmt for making available a preprint of Ref. 52 . 


\section{APPENDIX}

For two charge densities $\sigma_{1}$ and $\sigma_{2}$ distributed over surfaces $\Sigma_{1}$ and $\Sigma_{2}$, respectively, the FRCM integral equations are [18]:

$$
\begin{array}{ll}
\sigma_{1}=\frac{1}{4 \pi}\left(1-\frac{1}{\varepsilon_{\infty}}\right)\left[\frac{\partial \varphi}{\partial n}+\hat{S}_{11} \sigma_{1}+2 \pi \sigma_{1}+\hat{S}_{12} \sigma_{2}\right] & \left(r \in \Sigma_{1}\right) \\
\sigma_{2}=\frac{1}{4 \pi}\left(1-\frac{\varepsilon_{\infty}}{\varepsilon_{0}}\right)\left[\frac{\partial \varphi}{\partial n}+\hat{S}_{21} \sigma_{1}+\hat{S}_{22} \sigma_{2}+2 \pi \sigma_{2}\right] & \left(r \in \Sigma_{2}\right)
\end{array}
$$

Here $\frac{\partial}{\partial n}$ denotes the normal derivative relative to the corresponding surface and

$$
\varphi(\boldsymbol{r})=\int_{v_{1}} d^{3} \boldsymbol{r}^{\prime} \frac{\rho\left(\boldsymbol{r}^{\prime}\right)}{\left|\boldsymbol{r}-\boldsymbol{r}^{\prime}\right|}
$$

is the vacuum potential due to the solute charge $\rho(r)$. Surface integral operators $\hat{S}_{i j}$; $i, j=1,2$ are explicitly defined in [18]. The only comment required here is that cross terms $\hat{S}_{12} \sigma_{2}$ and $\hat{S}_{21} \sigma_{1}$ represent the interaction between $\sigma_{1}$ and $\sigma_{2}$.

The total response field is

$$
\Phi_{t o t}(\boldsymbol{r})=\Phi_{1}(\boldsymbol{r})+\Phi_{2}(\boldsymbol{r})
$$

where

$$
\begin{aligned}
& \Phi_{1}(\boldsymbol{r})=\int_{\Sigma_{1}} d^{2} \boldsymbol{r}^{\prime} \frac{\sigma_{1}\left(\boldsymbol{r}^{\prime}\right)}{\left|\boldsymbol{r}-\boldsymbol{r}^{\prime}\right|} \\
& \Phi_{2}(\boldsymbol{r})=\int_{\Sigma_{2}} d^{2} \boldsymbol{r}^{\prime} \frac{\sigma_{2}\left(\boldsymbol{r}^{\prime}\right)}{\left|\boldsymbol{r}-\boldsymbol{r}^{\prime}\right|}
\end{aligned}
$$

The inertial component of $\Phi_{t o t}(r)$ is found as the difference of total and inertialess fields:

$$
\Phi(\boldsymbol{r})=\Phi_{t o t}(\boldsymbol{r})+\Phi_{\infty}(\boldsymbol{r})
$$

where

$$
\Phi_{\infty}(\boldsymbol{r})=\int_{\Sigma_{1}} d^{2} \boldsymbol{r}^{\prime} \frac{\sigma_{\infty}\left(\boldsymbol{r}^{\prime}\right)}{\left|\boldsymbol{r}-\boldsymbol{r}^{\prime}\right|}
$$

The inertialess charge density $\sigma_{\infty}(r)$ is located on $\Sigma_{1}$ and defined in terms of the PCM-BKO equation with single surface $\Sigma_{1}$ : 


$$
\sigma_{\infty}=\frac{1}{4 \pi}\left(1-\frac{1}{\varepsilon_{\infty}}\right)\left[\frac{\partial \varphi}{\partial n}+\hat{S}_{11} \sigma_{\infty}+2 \pi \sigma_{\infty}\right]
$$

In general, $\sigma_{1}$ is composed of inertialess $\left(\sigma_{\infty}\right)$ and inertial $\left(\sigma_{1}-\sigma_{\infty}\right)$ components. One can see, however. that. provided the interaction term $\hat{S}_{12} \sigma_{2}$ can be neglected in the first Eq. (A1), then

$$
\begin{aligned}
& \sigma_{1}=\sigma_{\infty} \\
& \sigma_{2}=\sigma
\end{aligned}
$$

where $\sigma$ is the inertial charge density entirely located on $\Sigma_{2}$. This is an essence of so called "the intermediate uniform approximation" [18]. It becomes the exact procedure in spherically symmetric systems. where the explicit separation of inertialess and inertial contributions to the solvation energy is readily apparent (see Eq. (26)). Different versions and features of the uniform approximation, which extends to solutes with arbitrary shapes and charge distributions the simplifications inherent in spherically symmetric solutes, are discussed in [18] and [54].

\section{REFERENCES}

1. Agranovich V. M., and Ginsburg V. L., Spatial Dispersion in the Crystal Optics and the Theory of Exitons, New York: Interscience Publishers, Willey, 1973.

2. Ovchinnikov A. A.. and Ovchinnikova M. Ya., Soviet Phys. JETP 56. 1278 (1969).

3. Dogonadze R. R., Komyshev A. A., and Kuznetsov A. M., Theor. and Mathem. Phys. 15, 127 (1973), in Russian.

4. Dogonadze R. R., and Komyshev A. A., Phys. Stat. Sol. b53, 439 (1972)

5. Ulstrup J., Charge transfer processes in condensed media. // Lecture notes in chemistry, Berlin, Springer, 1979.

6. Wertheim M. S.. Ann. Rev. Phys. Chem. 30, 471 (1979).

7. Miertus S., Scrocco E.. and Tomasi J., Chem. Phys. 55, 117 (1981).

8. Tomasi J., and Persico M., Chem Rev. 94, 2027 (1994).

9. Constanciel R., Theor. Chim. Acta 69, 505 (1986).

10. Basilevsky M. V., and Chudinov G. E., Chem Phys. 157, 327, 345 (1991).

11. Basilevsky M. V., and Chudinov G. E., J. Mol. Structure (THEOCHEM) 260, 223 (1992).

12. Basilevsky M. V., Chudinov G. E., and Newton M. D., Chem. Phys. 179, 263 (1994).

13. Kim H. J.. and Hynes J. T., J. Chem. Phys 93, 5194 (1990).

14. Kim H. J., and Hynes J. T., J. Chem. Phys 96, 5088 (1992).

15. Bianco R., and Hynes J. T., J. Chem. Phys. 102, 7864, 7885 (1995).

16. Basilevsky M. V., and Chudinov G. E., Chem. Phys. 165, 213 (1992).

17. Hummer G., Pratt L. R., and Garcia A. E., J. Phys. Chem. A 102, 7885 (1998).

18. Basilevsky M. V., Rostov I. V., and Newton M. D., Chem. Phys. 232, 189 (1998); Newton M.D., Rostov I. V., and. Basilevsky M. V., Chem. Phys. 232, 201 (1998).

19. Morse P.M., and Feshbach H., Methods of Theoretical Physics, Part I, New York: McGraw-Hill, 1953.

20. Newton M. D., and Friedman H. L., J. Chem. Phys. 88, 4460 (1988). 
21. van der Zvan G., Hynes J. T., J. Phys. Chem. 89, 4181 (1985); Wolynes P.G., J. Chem. Phys. 86, 5133 (1987).

22. Simon J. D., Acc. Chem. Res. 21, 128 (1988); Baghi B., and Chandra A., Adv. Chem. Phys. 80, I (1991); Horng M., Gardecki J., Papazyan A., and Maroncelli M., J. Phys. Chem. 99, 17311 (1995).

23. Basilevsky M. V., Parsons D. F., and Vener M. V., J. Chem. Phys 108, 1103 (1998).

24. Song X., and Chandler D., J. Chem. Phys. 108, 2594 (1998).

25. Parsons D. F., Vener M. V., and Basilevsky M. V., J. Phys. Chem. A 103, 1171 (1999).

26. Basilevsky M. V., and Chudinov G. E., Mol. Phys. 65, 1121 (1988).

27. Grote R., and Hynes J. T., J. Chem. Phys. 74, 4465 (1981); 75, 2191 (1981).

28. Hanggi P., Talkner P., and Borkovec M., Rev. Mod. Phys. 62, 251 (1990).

29. Klamt A.. Schüürmann G., J. Chem. Soc. Perkin Trans. I1, No. 5, 799 (1993); Klamt A., J. Phys. Chem. 100, 3349 (1996).

30. Cramer C. J., and Truhlar D. J., J. Am. Chem. Soc 113, 8305 (1991).

31. Cramer C. J., and Truhlar D. J., Chem. Rev. 99, in press (1999).

32. Liu Y.-P., and Newton M. D)., J. Phys. Chem. 99, 12382 (1995).

33. Basilevsky M. V., Chudinov G. E., Rostov I. V., Liu Yi-Ping, and Newton M. D., J. Mol. Structure (THEOCHEM) 371, 191 (1996).

34. Basilevsky M. V., and Parsons D. F., J. Chem. Phys 105, 3734 (1996).

35. Raineri F. O., Resat H., Perng B.-C., Hirata F., and Friedman H. L., J. Chem. Phys. 100, 1477 (1994); Raineri F. O., Perng B.-C., and Friedman H. L., Chem. Phys. 183, 187 (1994).

36. Perng B.-C., Newton M. D., Raineri F. O., and Friedman H. L., J. Chem. Phys. 104, 7153, 7177 (1996).

37. Bevcridge D. L., and Schnuelle G. W., J. Phys. Chem 79, 2562 (1975); Abraham M. H., Lizli J., and Meszaros L., J. Chem. Phys. 70, 2491 (1979).

38. Dogonadze R. R., and Kornyshev A. A., J. Chem. Soc. Far. Trans. 2 70, 1121 (1974)

39 Kornyshev A. A, in The Chemical Physics of Solvation. Part A. / Eds. Dogonadze R. R., Kalman

E., Kornyshev A. A., Ulstrup J., Amsterdam: Elsevier, 1985, p. 77.

40. Basilevsky M. V., and Parsons D. F., J. Chem. Phys. 108, 9107, 9114 (1998).

41. Blum L., J. Chem. Phys., 61, 2129 (1974); Chan D. Y. C., Mitchell D. J., and Nikham B. W., J. Chem. Phys. 70, 2946 (1979).

42. Chandra A., and Bagchi B., J. Chem. Phys. 90, 1832 (1989); Atard P., Wei D., and Patey G. N., Chem. Phys. Lett., 172, 69 (1990); Fonseca T.. and Ladanyi B. M., J. Chem. Phys. 93, 8148 (1990); Fonseca T., and Ladanyi B. M., J. Phys. Chem 95, 2116 (1991); Raineri F. O., Zhou Y., and Friedman H. L., Chem. Phys. 152, 201 (1991); Raineri F. O., Resat H., and Friedman H. L., J. Chem. Phys. 96, 3068 (1992); Bopp P. A., Komyshev A. A., and Sutmann G., Phys. Rev. Lett., 76, 1280 (1996).

43. Kornyshev A. A., and Sutmann G., J. Chem. Phys., 104, 1524 (1996).

44. Kim H. J., J. Chem. Phys. 105, 6818 (1996); Jeon J., and Kim H. J., J. Chem. Phys. 106, 5979 (1997).

45. Komyshev A. A., Leikin S., and Sutmann G., Electrochim. Acta 42, 849 (1997).

46. Kumar K., Kurnikov I. V., Beratan D. N., Waldeck D. H., and Zimmt M. B., J. Phys. Chem. A 102, 5529 (1998).

47. Stewart J. J. P., J. Comput. Chem. 10, 221 (1989).

48. Marcus Y., Ion Sovation, New York: Wiley, 1985.

49. Miller J. R., Paulson B. P., Bal R, and Closs G. L., J. Phys. Chem. 99, 6923 (1995).

50. Matyshov D. V., Chem. Phys. 174, 199 (1993).

51. Elliott C. M., Derr D. L., Matyushov D. V., and Newton M. D., J. Am. Chem. Soc. 120, 11714 (1998).

52. Vath P., Zimmt M. B., Matyushov D. V., and Voth G., J. Phys. Chem. B, in press.

53. Tawa G. J., and Pratt L. R., J. Am. Chem. Soc. 117, 1625 (1995).

54. Kharkats Yu. 1., Kornyshev A. A., and Vorotyntsev M. A., J. Chem. Soc., Faraday Trans. II 72, $361(1976)$ 
